\title{
Circuit
}

Musiques contemporaines

\section{Micheline Coulombe Saint-Marcoux et Marcelle Deschênes : pionnières dans le sentier de la création électroacoustique Micheline Coulombe Saint-Marcoux and Marcelle Deschênes: Electroacoustic Pioneers}

\section{Marie-Thérèse Lefebvre}

Volume 19, numéro 1, 2009

Composer au féminin

URI : https://id.erudit.org/iderudit/019932ar

DOI : https://doi.org/10.7202/019932ar

Aller au sommaire du numéro

Éditeur(s)

Les Presses de l'Université de Montréal

ISSN

1183-1693 (imprimé)

1488-9692 (numérique)

Découvrir la revue

Citer cet article

Lefebvre, M.-T. (2009). Micheline Coulombe Saint-Marcoux et Marcelle Deschênes : pionnières dans le sentier de la création électroacoustique. Circuit, 19(1), 23-41. https://doi.org/10.7202/019932ar

\section{Résumé de l'article}

L'auteure tente de cerner les attitudes " masculines » et " féminines " de la création musicale des cinquante dernières années afin de faire ressortir la contribution de deux compositrices québécoises, Micheline Coulombe Saint-Marcoux et Marcelle Deschênes, au développement de la musique électroacoustique au Québec. Par une mise en contexte historique, l'auteure situe dans un premier temps le milieu dans lequel elles ont vécu leur enfance, leur adolescence, et leur formation musicale à Montréal et à Paris ; puis la manière dont elles ont traversé le discours dominant des années 1970, pour finalement aborder leur rôle de pionnières. Parce qu'elles voulaient sortir des sentiers battus, elles ont compris que les nouveaux outils technologiques pourraient leur servir à exprimer musicalement une nouvelle sensibilité artistique. En ce sens, elles furent les « sherpas » du milieu électroacoustique au Québec. 


\section{Micheline Coulombe Saint-Marcoux et Marcelle Deschênes : pionnières dans le sentier de la création électroacoustique}

Marie-Thérèse Lefebvre

\section{Introduction}

Lorsque le rédacteur en chef de la revue Circuit m'a proposé d'écrire un article sur Micheline Coulombe et sur Marcelle Deschênes, dans le cadre d'un numéro qui serait consacré à la présence des femmes dans le milieu musical, j’ai beaucoup hésité. Pour deux raisons.

D'une part, je ne voyais aucun intérêt à ressasser les sources secondaires pour répéter en d'autres mots ce qui avait déjà été dit sur leur formation et leur musique. Par ailleurs, il me semblait difficile d'apporter un éclairage nouveau visant à souligner leur importance dans l'histoire sans d'abord retourner aux sources primaires que sont les archives qui, dans les deux cas, sont encore du domaine privé. Chacune a tenu un journal personnel et a entretenu une correspondance dont la consultation (impossible pour l'instant) permettrait sans doute d'en savoir un peu plus sur leur réseau professionnel et sur la manière dont elles ont surmonté les difficultés du métier de compositeur dans les années pures et dures de la vague structuraliste des années 1960 et 1970, les «années glorieuses» disaient certains'.

D'autre part, je m'interrogeais sur la pertinence, en 2008, d'isoler les compositrices dans un numéro spécial qui leur serait consacré. Ces interrogations identitaires lancinantes, «Y a-t-il une musique québécoise ?» et «Existe-t-il une musique au féminin ?3» me semblaient obsolètes. Du côté de la littérature, du théâtre, du cinéma et des arts visuels, ces questions ne se posent
1. Expression inventée par l'économiste français Jean Fourastié dans Les Trente Glorieuses ou la révolution invisible de 1946-1975 (Paris, Fayard, 1979).

2. Nattiez, 1987

3. Green et Ravet, 2005 
4. Lefebvre, 2004, particulièrement les pages 226 à 231 .

5. Chénard, 2008, p. 52. Dans le texte original, les noms cités par l'auteure pour chacune des disciplines sont formulés dans un second paragraphe. Nous les avons intégrés dans les parenthèses pour en faciliter la lecture. 6. Gann, article dans ce numéro. plus: ces expressions artistiques se sont, depuis longtemps déjà, affranchies. Or, dans le cas de la musique de création, la question la plus importante n'est-elle pas de vérifier en premier lieu si cette production de nos compositeurs et compositrices est reconnue comme partie intégrante de la culture québécoise? Car, si le statut professionnel de compositeur a émergé dans les années 1930, comme j'ai tenté de le démontrer4, il ne paraît pas avoir réussi, à ce jour, à s'institutionnaliser, c'est-à-dire à être reconnu de manière plus large, en dehors du milieu proprement musical. Très peu d'intellectuels intéressés à l'histoire culturelle ou de hauts fonctionnaires qui représentent le Québec à l'étranger, entre autres, peuvent spontanément aujourd'hui citer quelques noms de compositeurs/compositrices (morts ou vivants). J'en veux pour preuve cette affirmation de la déléguée générale du Québec à Atlanta lors d'un récent colloque sur le Québec:

Plus que toute autre forme d'expression, les arts et la culture ont réussi à traduire les diverses métamorphoses de l'évolution de la nation québécoise et du peuple. La culture se distingue [...] dans plusieurs domaines: le cinéma (Claude Jutras et Denis Arcand), la danse moderne et classique (La La La Human Steps et les Grands Ballets Canadiens), la musique contemporaine et classique (Gilles Vigneault, Leonard Cohen, Arcade Fire et Ariane Moffat; les Violons du Roy, I Musici, Louis Lortie et l'Orchestre symphonique de Montréal), le théâtre et les arts de la scène (Robert Lepage, Wajdi Mouawad et le Théâtre du Nouveau-Monde), la littérature (Anne Hébert, Michel Tremblay et Dany Laferrière), les arts du cirque (Le Cirque du Soleil), la peinture (Jean-Paul Riopelle et Marc-Aurèle Fortin), le design de mode (Marie Saint-Pierre) $[\ldots]^{5}$.

Malgré tous les efforts que déploie le milieu, il reste donc beaucoup de travail à accomplir pour que l'information atteigne un public plus large. J'y joins ici ma modeste contribution. L'article du critique américain Kyle Gann ${ }^{6}$ m'a particulièrement intéressée car il propose une lecture différente de la création au féminin. Selon cet auteur, il ne s'agit plus d'isoler les compositrices ou d'étudier ce qui est distinctement féminin dans leur musique, mais plutôt d'analyser les attitudes «masculines» et «féminines» de la création musicale des cinquante dernières années afin de faire ressortir leur apport au changement de paradigme qui caractérise le langage musical postmoderne depuis le début de la décennie des années 1980.

C'est donc par l'analyse du contexte historique que je propose d'étudier dans un premier temps le milieu dans lequel Micheline Coulombe SaintMarcoux et Marcelle Deschênes ont vécu leur enfance, leur adolescence, et leur formation musicale; en second lieu, leurs études à Paris; puis la manière dont elles ont traversé le discours dominant des années 1970, pour finalement 
aborder leur effort de défrichage du terrain et leur contribution au renouveau du langage musical au début des années 1980.

\section{Tout se joue avant vingt ans}

Aucune notice biographique de ces deux compositrices n'offre d'information avant leur arrivée à Montréal au début des années 1960 alors qu’elles sont dans la jeune vingtaine. Or, on le sait, la formation de la personnalité d'un individu et les rêves d'avenir qui motiveront les futures décisions se cristallisent avant vingt ans. Lorsqu'elles entrent dans les classes de composition de Claude Champagne et de Jean Papineau-Couture au début des années 1960, Micheline et Marcelle portent déjà en elles un bagage de vie qu'il faut connaître pour comprendre la suite de leur cheminement.

Micheline Coulombe voit le jour le 9 août 1938 au Saguenay-Lac-Saint-Jean dans le petit village de Notre-Dame-de-la-Doré (aujourd'hui La Doré), tout près de Saint-Félicien7. Elle est élevée dans un milieu relativement aisé (son père Philippe est un entrepreneur doué dans l'exploitation forestière et un commerçant averti), dans une famille de musiciens amateurs (on y aime à chanter) et par une mère courageuse qui a eu 13 enfants (dont 7 vivants) et soucieuse de donner une éducation aussi large que possible à ses filles. Micheline fréquente l'école du village et y termine une $10^{\mathrm{e}}$ année, après quoi, elle s'inscrit, pensionnaire durant trois ans, à l'École normale de Roberval, tenue par les Ursulines. On la dit réservée et solitaire. C'est à cet endroit qu'elle suit ses premiers cours de piano et qu'elle s'adonne à quelques essais de composition. Sa professeure, Mère de la Trinité, l'encourage à rencontrer le compositeur et ethnomusicologue François Brassard, résidant à Jonquières, qui fut l'élève de Claude Champagne dans les années 1930. Ce qu'elle fera entre 1955 et 1958 tout en travaillant comme institutrice à l'école primaire de son village natal. Constatant la forte personnalité musicale qui se dégage des premiers travaux, Brassard lui recommande de poursuivre sa formation à Montréal à l'École Vincent-d'Indy, considérée alors comme une institution prestigieuse. Elle quitte définitivement la région du Saguenay pour s'établir à Montréal où elle partagera un appartement avec l'une de ses sœurs, infirmière. Craignant de ne pouvoir satisfaire aux exigences d'admission de cette école, Micheline s'inscrit d'abord à l'Institut Cardinal-Léger pour perfectionner ses connaissances. Elle est admise deux ans plus tard à l'École Vincent-d'Indy où elle obtient un diplôme en piano et le prix Maurice-Ravel pour la composition en 1962, après avoir suivi les cours de piano d'Yvonne Hubert et d'écriture musicale avec Champagne. Sachant qu'elle ne fera pas carrière comme pianiste, ce dernier l'incite à se diriger vers le Conservatoire de musique du Québec à Montréal.
7. Nous remercions chaleureusement Monsieur Jacques Saint-Marcoux de nous avoir transmis plusieurs informations inédites sur divers aspects de la vie et de la carrière de son épouse. 
8. Boivin, 2001, p. 75-90. 9. Coulombe Saint-Marcoux, 1968 ,
p. $13-16$.
Il lui suggère de rencontrer son ancien élève, le compositeur Clermont Pépin qui la prend sous son aile. Avec deux collègues inscrits au même programme, Marc Fortier et François Dompierre, elle suit la classe d'analyse de Gilles Tremblay et les cours d'écriture de Françoise Aubut, pédagogue remarquable (mais impitoyable dans l'évaluation des travaux des étudiants), qui l'encouragera à présenter son dossier au Prix d'Europe, qu'elle obtient en 1967 après l'attribution d'un premier prix en composition du Conservatoire. Elle a composé quelques pièces pour piano et pour voix, une sonate pour flûte et piano, un quatuor à cordes et deux œuvres pour orchestre utilisant les ondes Martenot, Vent et Modulaires.

Un parcours donc relativement traditionnel, bien encadré, sage et conforme, qui aurait pu la conduire dans la voie toute tracée d'une formation supérieure semblable au Conservatoire national de musique de Paris. Mais, comme bien des femmes des années 1960, elle cherche maintenant à tourner le dos à cette première formation, à se libérer d'un carcan, à sortir des sentiers battus, à explorer de nouveaux territoires. Elle a vu et entendu les environnements sonores de certains pavillons de l'Exposition universelle de Montréal ${ }^{8}$, dont le polytope du pavillon français créé par Iannis Xenakis qu'elle rencontre en décembre 1967 lors d'une conférence de ce dernier au Conservatoire. Il lui suggère d'approfondir certains concepts mathématiques qui pourraient lui servir à imaginer de nouvelles structures musicales. Constatant son besoin de «changer d'air », il lui recommande de joindre le Groupe de recherches musicales (GRM) alors dirigé par Pierre Schaeffer à Paris.

Juste avant de partir, elle signe son premier article dans lequel elle affirme ses positions sur le fait d'être compositrice (qui lui vaudra plus tard un certain boycottage des féministes), sur les programmes de formation, sur l'espoir que les œuvres des jeunes compositeurs puissent être entendues:

Devenir compositeur, c'est choisir une voie parsemée de tels obstacles, telles embûches, que le fait d'être une femme n'en ajoute pas davantage. [...] L'enseignement n'est pas toujours très adéquat. On insiste trop sur l'étude de systèmes périmés [...] qui ne répondent plus aux besoins actuels d'expression musicale. [...] On admet facilement $[. .$.$] que la littérature se fait chaque jour. On vous cite aisément [des$ noms d'auteurs]. En musique, tout semble figé. Qui peut citer des compositeurs québécois ?9

Nous sommes en mai 1968.

Marcelle Deschênes naît le 2 mars 1939 dans le petit village de Price d’à peine quelque mille habitants, situé entre Métis et Mont-Joli. Si elle n’a que peu de souvenirs d'un père souvent absent, elle a pour sa mère une profonde admiration. Aldégonde Deschênes (1910-1996) est en effet une femme plus 
grande que nature qui, tout en élevant ses six enfants, sera enseignante à l'école primaire de Price durant plus de 35 ans $^{10}$. D'une imagination débordante, elle initie les jeunes élèves à la poésie et à l'écriture par la voie de la création plutôt que par l'application sèche des règles grammaticales. Elle dessine, écrit des contes et des nouvelles ${ }^{11}$ et entretiendra une longue correspondance avec sa fille lorsque cette dernière étudiera à Paris. Dès son jeune âge, Marcelle, encouragée par sa mère, s'essaie à la poésie, expérimente la photographie et suit des cours de piano. Elle participe aux examens de l'Académie de musique devant Edwin Bélanger et Omer Létourneau, étudie à l'Institut familial de Rimouski durant deux ans et termine ses premières études musicales à l'École supérieure de musique de Nicolet. Pour tromper son isolement durant cette période passée à Price et dans divers couvents, elle développe un réseau de plusieurs correspondants internationaux choisis à partir d'une revue parisienne pour les jeunes, Lisette, à laquelle l'Institut familial était abonné.

Elle quitte définitivement Price à l'âge de 17 ans et, après avoir travaillé comme directrice musicale et pianiste au poste CKBL de Matane, elle s'installe à Montréal en 1962 où elle gagne sa vie comme pianiste à divers endroits, clubs, festivals, et aux Grands Ballets Canadiens. C'est à cette époque qu'elle fait la connaissance du poète Raoul Duguay. Elle s'inscrit l'année suivante à la Faculté de musique de l'Université de Montréal comme étudiante libre ${ }^{12}$ et obtient un baccalauréat, puis une licence en musique. On la dit rebelle, inclassable. Elle s'ingénue à s'amuser avec les procédés d'écriture, traditionnels ou sériels, et à rechercher un langage qui prend sa source dans des modèles ou structures évoquant les antagonismes et les mouvements contraires. Elle écrit quelques pièces instrumentales dans cet esprit provocateur: une sonate pour piano, composée en 1966 selon les règles formelles pour satisfaire aux exigences de la classe de Jean Papineau-Couture, et, l'année suivante, la même pièce mais avec un nouveau titre, $1^{1 / 2}{ }_{2}$, adaptée cette fois aux règles de l'écriture sérielle enseignées par Serge Garant dans sa classe d'analyse. Après une tentative avortée, faute de moyens techniques, de produire une pièce mixte, À la désespérade, et après avoir essuyé un refus pour son projet Cri-Cri pour la manière dont elle traite la voix, pour son contenu extramusical (textes de Che Guevara) et son aspect multidisciplinaire'3 ${ }^{13}$ elle présente $\mathrm{VOZ}$, cantate mitrailleuse, musique vocale inspirée du Che et dédiée aux causes révolutionnaires de l'époque. Après avoir découvert les textes de Paul Klee, elle écrit $7+7+7^{+} 7^{+}$, pour 15 instruments, un essai sur la transposition du visuel au sonore écrit selon les techniques sérielles, mais, dit Marcelle Deschênes avec humour, «avec l'ajout d'un diabolus in
10. Le comité du patrimoine de Price a fondé en 2007 un prix littéraire pour les jeunes qui porte son nom.

11. Elle reçoit en 1988 le prix Grand cœur Canada.

12. Sans le baccalauréat classique, elle ne peut être admise à un programme régulier. Elle suit quand même tous les cours du programme.

13. Ce projet d'une théâtralisation de la création de l'univers sous forme d'opposition entre le cri à la vie et le cri à la mort sera éventuellement intégré dans OPERAaaaAH en 1983. 
14. La compositrice fait allusion ici à l'introduction de couleurs dans la notation et de dessins stimuli comme blocs aléatoires d'improvisation dirigée (indication de réservoirs d'objets et de paramètres). Ce "diabolus in musica" est déjà présent dans VOZ.

15. Archives personnelles de Marcelle Deschênes.

16. On y présentait Zyklus et Klavierstucke 7 et 11 interprétées par David Tudor et Max Neuhaus, suivies de Refrain interprétée par Serge Garant, Gilles Tremblay et Guy Lachapelle.

17. Marcelle Deschênes commente ainsi cette rencontre: "Ce qui m'avait marquée dans ce qu'il avait analysé, c'était les partitions incluant des images et des graphismes, de la couleur sur les sons, de la spatialisation en direct, de l'amplification, une pensée électroacoustique appliquée aux instruments et aux voix. Tout était nouveau pour moi et correspondait davantage à ma sensibilité que des structures uniquement abstraites. Puisque ça se faisait ailleurs dans le monde, je pouvais le faire aussi. C'était ma première liberté, l'intégration de l'aléatoire et de l'improvisation dans une structure sérielle hyperstricte" (entrevue avec l'auteure, 7 août 2008)

Rappelons qu'en 1963 Herman Sabbe avait participé à la fondation du Groupe Spectra rattaché à l'IPEM (Institut de psychoacoustique et de musique électronique) de l'Université de Gand. C'est à ce studio que Micheline Coulombe Saint-Marcoux a réalisé les bandes de Miroirs (1975) et Regards (1978). musica ${ }^{14} »$. Son professeur Serge Garant lui écrit ce commentaire : «La transposition des éléments visuels est excellente, bien que ce genre de référence extramusicale m'inquiète un peu. J'ai peut-être tort, mais puisque cela prête à discussion, c'est que c'est vivant, donc très bien ${ }^{15}$.»

Trois événements vont la propulser vers un sentier inconnu : l'entrevue de Karlheinz Stockhausen avec Maryvonne Kendergi en janvier 1964 suivie d'un concert de ses œuvres à la salle Saint-Sulpice ${ }^{16}$; la forte impression qu'elle reçoit en 1967 des conférences sur la musique contemporaine présentées par le musicologue belge Hermann Sabbe ${ }^{17}$ au pavillon des Jeunesses Musicales, et la découverte du fameux théâtre pluridisciplinaire La Lanterna Magika du metteur en scène Alfréd Radok et du scénographe Josef Svoboda présenté au pavillon de Tchékoslovaquie, durant l'Exposition universelle de Montréal.

L'imagination est en marche. Récipiendaire de quatre premiers prix en piano du Festival de musique du Québec (section Bas-Saint-Laurent), de la Médaille du Lieutenant-gouverneur du Québec pour l'excellence de ses études de baccalauréat, et de deux premiers prix au Concours de photographie de la Presse internationale, Marcelle Deschênes s'installe à Paris, munie de trois bourses de perfectionnement pour artiste, provenant des gouvernements de France, du Canada et du Québec. Nous sommes en mai 1968.

\section{Prendre le large}

Jusqu'ici, cette première formation dans les institutions musicales montréalaises a lieu à un moment où la présence des femmes comme étudiantes est encore largement majoritaire (quoique peut-être un peu moins au Conservatoire), car c'est l'époque où les religieuses viennent compléter leur formation universitaire afin de s'adapter aux nouveaux programmes d'enseignement imposés par le rapport Parent. Les perspectives de carrière en composition pour les jeunes étudiantes laïques reposent cependant sur les modèles masculins que représentent Papineau-Couture, Pépin et Tremblay, professeurs réguliers et compositeurs de musique instrumentale dont les œuvres sont jouées par des organismes reconnus. Elles n'ont par contre aucune connaissance du mode de fonctionnement du milieu musical proprement dit, de son réseau, de ses chapelles, de ses mécanismes de production et de diffusion. Elles feront cet apprentissage au moment de leur retour d'études.

Dans ce contexte, la décision de se diriger vers le GRM et la musique électroacoustique (nommée «concrète » par Schaeffer et «électronique » par Stockhausen, à l'époque) peut donc sembler curieuse. Car s'il est vrai que ces nouvelles approches technologiques étaient connues au Québec, entre autres, par Serge Garant, Maurice Blackburn et Gilles Tremblay, nous 
n'avons aucune preuve que les deux jeunes compositrices aient eu connaissance de la venue de Stockhausen à Montréal en 1958 et de Messiaen en 1962, ou qu'elles aient participé en août 1960 soit à la présentation d'œuvres représentatives de ce courant par Gilles Tremblay au camp musical d'Orford ou encore à la Semaine internationale de musique actuelle organisée en août 1961 par Pierre Mercure durant laquelle des œuvres électroacoustiques furent présentées $^{18}$. L'animatrice de l'émission Festivals européens et cofondatrice de la Société de musique contemporaine du Québec, Maryvonne Kendergi, connaissait Schaeffer depuis 1950. Elle en a peut-être fait mention durant son émission Festivals européens diffusée entre 1957 et 1963, mais ce n'est qu'en novembre 1969 (les deux compositrices étant alors à Paris) qu'elle organise la Semaine Schaeffer (premier musialogue) en présence du compositeur. Ajoutons qu'entre septembre 1961 et mai 1967, date de l'ouverture de l'Exposition universelle, fort peu d'œuvres électroacoustiques étaient diffusées à la radio ou en concert. Seules 4 des 85 œuvres québécoises composées durant cette période (1961-1966) ont utilisé des procédés électroacoustiques, et ces pièces étaient de Pierre Mercure.

C'est donc beaucoup plus sous l'impulsion de la recherche d'une personnalité musicale qui leur soit propre, hors des techniques de l'écriture sérielle enseignées de manière tout aussi codée (enseignement que Marcelle Deschênes nommait «le temps des corsets») que celles de l'écriture harmonique tonale, qu'elles ont orienté leurs études vers le GRM. En cela, dois-je ajouter que cet élan irrépressible vers le changement prenait ses racines dans un mouvement social très profond où les rôles traditionnels dans lesquels la société avait enchâssé les femmes étaient en train de basculer de manière radicale au sein de cette même génération. Nous étions les purs produits de Simone de Beauvoir et de Betty Freidan et tout embrigadement dans un mouvement quelconque (même féministe pour certaines) en éloignait plusieurs. Nous cherchions de l'oxygène...

Micheline Coulombe épouse Jacques Saint-Marcoux, professeur de littérature, en 1965. Ils s'envolent vers Paris à l'été 1968. Elle s'inscrit au programme du GRM qui dure trois ans. Ce programme recevait une soixantaine d'étudiants pour n'en garder que quelques-uns au début de la troisième année. Elle suit les cours théoriques de Pierre Schaeffer au Conservatoire national de musique durant la première année, joint le GRM pour y faire l'apprentissage technique en studio avec Guy Reibel, aborder les relations son-image avec Bernard Parmegiani, et réfléchir aux aspects poétiques du phénomène sonore avec François Bayle. Elle produit trois bandes magnétiques, Arksalalartoq, Contrastances et Moustières, et participe à une œuvre
18. Beaucage, 2008, p. 19-23. 
19. Le compositeur canadien, mars 1974.

20. Jean-Pierre Guézec avait été nommé en 1969 responsable de la classe d'analyse au Conservatoire national de Paris en remplacement d'Olivier Messiaen. II meurt d'une crise cardiaque foudroyante le 9 mars 1971 à l'âge de 35 ans.

21. Le compositeur canadien, mars 1974.

22. Elle n'aura produit, au cours de sa carrière, que six œuvres pour bande. Outre les quatre pièces mentionnées plus haut, elle compose Zones en 1972 et Constellation I en 1981, gravées sur Empreintes DIGITALes, IMED 0159.

23. Elle fera d'ailleurs un bref séjour à Montréal durant cet été pour assister aux répétitions de la pièce.

24. François Bayle avait organisé dans le cadre de ce festival un événement "Musiques éclatées" où 10 groupes formés de quatre stagiaires devaient proposer une œuvre collective. Selon le critique du journal Le Monde (13 août 1969), Robert Siohan, le résultat de cette expérience fut cependant mitigé (archives de Marcelle Deschênes). collective du GRM au Festival d'Avignon l'année suivante. Boursière du Conseil des Arts du Canada en 1969, elle s'inscrit à la classe de Gilbert Amy dont elle dit qu'il poussait le souci de l'organisation jusqu'à la limite, «une approche qui me paralysait ${ }^{19} »$. Elle écrit sous sa direction une pièce pour piano, Assemblages. Elle rencontre le compositeur Jean-Pierre Guézec²o dont «les cours ont été une sorte de soulagement car son contact facile et ouvert à la discussion m'a beaucoup stimulée $\mathrm{e}^{21}$. » Au cours de la troisième année, elle organise une tournée de concerts avec le GIMEP (Groupe international de musique électroacoustique de Paris) qu'elle avait fondé l'année précédente avec Eduardo Bertola (Argentine), Dieter Kaufmann (Autriche), Pierre Boëswillwald (France), Joanna Bruzdowicz (Pologne) et Jacques Lejeune (France), afin de faire connaître les nouvelles œuvres des membres. Dans le cadre de ces tournées, elle diffuse Bernavir. Parallèlement à ces activités, elle suit quelques cours d'analyse dans la classe d'Olivier Messiaen et fera un bref arrêt chez Nadia Boulanger, une rencontre qui ne durera que trois heures. Elle sortira de cette entrevue complètement rebutée par l'autoritarisme de cette célèbre pédagogue.

Il est important de préciser ici (et nous y reviendrons) que Micheline Coulombe Saint-Marcoux n'a jamais eu l'idée, en s'inscrivant au GRM, de naviguer uniquement dans les eaux de l'écriture électroacoustique et d'y faire carrière ${ }^{22}$. Il s'agissait pour elle de s'imprégner de ce nouveau langage pour ensuite tenter de l'intégrer soit à des œuvres mixtes, soit à en appliquer les concepts à des œuvres purement instrumentales. Tel est le sens de Hétéromorphie pour orchestre, une œuvre commandée par l'Orchestre symphonique de Montréal et écrite à Paris, qui sera créée en $1970^{23}$. Elle revient définitivement au Québec à l'été 1971 et obtient un poste de professeur au Conservatoire de musique à Montréal.

Marcelle Deschênes s'installe à Paris en septembre 1968 pour une durée de trois ans. Elle suit durant quelques mois les cours de Pierre Schaeffer et joint le GRM pour l'apprentissage technique auprès de François Bayle, Henri Chiarucci et Guy Reibel. Elle participe au Festival d'Avignon ${ }^{24}$ dans une œuvre collective, Chut, avec Micheline Coulombe Saint-Marcoux, Pierre Bernard et Richard Grégoire qu'elle avait connu à Montréal dans les classes de Serge Garant l'année précédente. Mais, déçue de ne trouver en ces lieux que des discussions théoriques sans véritable formation pratique intensive des nouvelles technologies, elle bifurque et suit les cours d'analyse d'Olivier Alain à l'école César-Franck et étudie la composition avec Gilbert Amy. Elle consacre la dernière année à des séminaires en ethnomusicologie donnés par Claudie-Marcelle Dubois à l'École Pratique des Hautes Études 
et assiste aux cours sur les musiques extraoccidentales de Claude Laloom à l'Université Paris VIII (Centre expérimental de Vincennes). Une formation éclatée donc qui la conduit de plus en plus vers des expressions musicales pluridisciplinaires. Durant ce séjour, elle compose Encore et toujours ce si25, pour hautbois et bande, et Talilalilalilalarequiem, une musique mixte qui s'éloigne de plus en plus de l'écriture sérielle et puise son modèle formel dans la structure de l'énergie nucléaire, métaphore de la rencontre de forces antinomiques (fusion d'atomes) d'une même réalité. L'œuvre joue sur des principes de lenteur, de sons démesurément longs, et les sons électroacoustiques sont utilisés comme une extension des sons acoustiques. La difficulté d'intégration de ces deux mondes la convainc d'une chose: son médium sera désormais uniquement celui de l'électroacoustique. À son retour au Québec, elle multipliera les expériences de recherche et élaborera des outils pédagogiques spécifiques qui la conduiront, dix ans plus tard, à fonder à l'Université de Montréal un programme de composition électroacoustique aux trois cycles universitaires.

\section{Maintenir le cap contre vents et marées}

Le milieu musical, ici comme ailleurs, est constitué de réseaux relativement hermétiques et si l'Année internationale de la femme en 1975 a permis de libérer la parole féminine qui a progressivement trouver «les mots qu'il fallait pour dire ${ }^{26}$ », elle n'en a pas pour autant favorisé le dialogue entre hommes et femmes. Car plus les femmes s'exprimaient, ici à travers la musique, plus les hommes se muraient dans des attitudes, des sous-entendus et des non-dits devant cette production artistique au féminin.

Deux milieux contrôlés par ces derniers ont été particulièrement résistants à l'arrivée des femmes dans leurs rangs dans les années 1970: celui de l'enseignement de la composition et celui des organismes de diffusion. Mais comment parler objectivement de ces tensions? D’une part, faire état de cette résistance masculine à partir de sources fiables est pratiquement impossible car celle-ci ne s'est jamais exprimée au grand jour, et d'autre part, les compositrices qui étaient à ce moment en formation ou en début de carrière ont hésité à exprimer ouvertement les difficultés qu'elles ressentaient à faire valoir leur travail professionnel. Tout se passait dans le chuchotement. En rendre compte relève nécessairement de la perception subjective de celui ou de celle qui a vécu ou a été témoin de ces attitudes. Tentons malgré tout d'exposer quelques faits et d'analyser rétrospectivement les enjeux.

Jusqu'au début des années 1970, la formation des compositeurs était assujettie à l'étude intensive des techniques d'écriture (harmonie, contrepoint,
25. En référence à cette note "si ", manquante dans le thème de onze sons de L'Offrande musicale de JeanSébastien Bach et à cette même note sur laquelle Berg construit cet immense crescendo annonçant la mort de Marie dans Wozzeck.

26. Allusion au titre du roman de Marie Cardinal Les mots pour le dire (Paris, Grasset, 1975). 
27. Conrad Letendre, Gabriel Cusson, Françoise Aubut et Massimo Rossi sont parmi les plus connus.

28. La plupart sont en contact avec les compositeurs européens dont plusieurs viendront à Montréal, créant ainsi un important réseau international dont pourront bénéficier les étudiants. fugue) enseignées principalement par des organistes ${ }^{27}$, gardiens de la tradition. Des changements importants ont lieu sous les mandats de Clermont Pépin et de Jean Papineau-Couture, directeurs du Conservatoire et de la Faculté de musique, qui procèdent à l'engagement de nouveaux compositeurs formés aux techniques modernes de composition ${ }^{28}$ et à une révision des programmes de formation. Deux idéologies s'affrontent alors: soit celle de former des étudiants rompus aux principes de l'écriture scolastique leur permettant de s'adapter aux diverses possibilités d'application de la composition ou soit celle de former des candidats à la maîtrise des nouveaux outils d'écriture, sériels et post-sériels, de manière à ce qu'ils puissent intégrer les rangs de la musique contemporaine d'avant-garde et y tracer leur marque personnelle.

Ces discussions d'un monde masculin ont abouti à la formation de deux programmes distincts, mais quelques professeurs manifestent un certain malaise devant la polarisation des tendances extrêmes de ces programmes, souhaitant pour leurs propres étudiants une connaissance approfondie de l'analyse des œuvres marquantes de l'histoire, une maîtrise des techniques d'écriture ainsi qu'une plus grande liberté dans le choix du langage, en fonction de la personnalité du candidat et du chemin qu'il entend suivre pour exprimer son univers musical. C'est vers cette école de pensée, représentée par Gilles Tremblay et André Prévost, puis par José Évangélista et Denis Gougeon, entre autres, que se dirigeront la plupart des futures compositrices dans les années 1980. Car, toujours pour les mêmes raisons qui ont farouchement guidé de nombreuses femmes dans leur choix de vie et de carrière à cette époque, elles opteront pour une formation souple où elles n'auront pas à subir des «maîtres» ou des règles d'écriture hors desquelles «point de salut».

Quant à l'introduction d'un programme spécifique à la musique électroacoustique, l'idée plane déjà et quelques essais prennent forme, mais les coûts en équipements techniques nécessaires à sa réalisation freinent d'autant plus toute prise décision que peu de compositeurs de musique instrumentale croient à l'avenir de ce médium. Il faudra attendre la force de conviction de deux compositrices et la convergence d'un intérêt partagé par une autre génération de compositeurs pour voir naître ce nouveau programme au début des années quatre-vingts.

Une autre difficulté que doivent affronter les compositrices dans les années 1970 est celle de convaincre les sociétés de production de leur commander des œuvres et de les faire jouer. Ce milieu possède, comme nous l'avons dit, son réseau et ses chapelles et, de manière générale, les directeurs artistiques 
et la plupart des membres des conseils d'administration sont des hommes. Aux organismes déjà existants, comme l'Orchestre symphonique de Montréal (OSM) ou les Jeunesses musicales du Canada, qui commandaient à l'occasion des œuvres à des compositeurs québécois, 34 nouveaux organismes sont apparus entre la fondation de la Société de musique contemporaine du Québec (SMCQ) en 1966 et la création des Événements du Neuf en 1979. Trois d'entre eux se sont consacrés à la diffusion de musique électroacoustique: MetaMusic ${ }^{29}$, GIMEL ${ }^{30}$ et le Groupe Sonde ${ }^{31}$. Les 31 compagnies restantes se consacraient au répertoire européen. C'est donc dire que la SMCQ a été pratiquement le seul lieu (en dehors des conservatoires et des universités) où les compositrices pouvaient espérer être jouées.

C'est dans ce contexte que Micheline Coulombe Saint-Marcoux construit sa carrière à Montréal. Au Conservatoire, on lui propose, dans le cadre des nouveaux programmes, un poste au niveau du cegep où elle enseigne les cours de littérature et langages musicaux. Avec l'appui d'Otto Joachim ${ }^{32}$ et de Gilles Tremblay, elle tente de mettre sur pied une cellule électroacoustique aux cycles supérieurs, mais ces démarches s'avèrent difficiles sous la direction de l'organiste Raymond Daveluy33. Elle poursuit son enseignement jusqu'en 1979 où elle doit se retirer pour combattre un premier cancer. À son retour à l'enseignement en 1983 , elle prendra en charge une classe de composition électroacoustique. C'est à ce moment, m'a confié Isabelle Panneton, qu'elle la rencontre pour la première fois, et c'est par la qualité de son écoute attentive auprès des étudiants, ajoute-t-elle, que la compositrice lui infusera la confiance nécessaire pour poursuivre l'aventure de la création ${ }^{34}$.

L'engagement de Micheline Coulombe Saint-Marcoux va cependant bien au-delà du cadre restreint de l'enseignement. Devant le peu de visibilité accordée aux créateurs, elle deviendra une voix pour la jeune musique contemporaine. À ce titre, elle entreprend la réalisation de plusieurs projets dans le but de faire connaître la musique des compositeurs du Québec.

Elle fonde en 1971 le groupe Polycousmie avec les percussionnistes Pierre Béluse, Guy Lachapelle et Robert Leroux. Ils créeront deux œuvres collectives, Épisodie avec le groupe de danse de la Nouvel'Aire et, à la demande de Jean Laurendeau, Séquences, pour deux ondistes et un percussionniste, dans le cadre des Nocturnales de la Faculté de musique de l'Université de Montréal. Ces œuvres aléatoires faisaient entendre des sons manipulés et amplifiés par des microphones reliés aux poignets des percussionnistes. En 1972, elle organise, avec la collaboration de Jacques Thériault, le «Carrefour électroacoustique », un événement qui officialise en quelque sorte l'entrée de la musique électroacoustique sur la scène québécoise ${ }^{35}$. Elle réitère son
29. Fondé en 1971 par Kevin Austin à l'Université Concordia et consacré à la musique électroacoustique improvisée. Cesse ses activités en 1978.

30. GIMEL (Groupe d'interprétation de musique électroacoustique de Laval), fondé par Nil Parent, directeur du SMEUL (Studio de musique électroacoustique de l'Université Laval) en janvier 1974; l'organisme cesse ses activités en janvier 1977. Parmi les membres permanents, outre son directeur, on comptait Marcelle Deschênes, Gisèle Ricard et Jean Piché.

31. Fondé par Charles de Mestral en 1975, le groupe se spécialise dans la performance et l'installation sonore. Cesse ses activités en 1987.

\section{Professeur de musique de} chambre au McGill Conservatory et au Conservatoire de musique du Québec, Otto Joachim avait créé son propre studio électroacoustique dès le milieu des années 1950.

33. Ce n'est qu'en 1979, alors qu'elle est déjà malade, qu'Yves Daoust, de retour d'un séjour d'études au sein du Groupe de musique expérimentale de Bourges, réalisera ce projet. Responsable d'un cours de technique électroacoustique aux cycles supérieurs en 1981, il la remplace en 1986, mais ce n'est qu'en 1994, après avoir convaincu l'administration, qu'il réalisera la création d'un véritable programme en composition électroacoustique au Conservatoire de Montréal.

34. Conversation avec la compositrice, le 23 juillet 2008.

35. On a présenté, du 21 au 23 avril, à la Galerie Trois (dirigée par Jeanne Renaud et Ed Kostener) dans le VieuxMontréal, 25 œuvres "acousmatiques" dont 20 de compositeurs canadiens et celles des cinq membres du GIMEP qu'elle avait fondé à Paris quelques années auparavant. La troupe de danse Nouvel'Aire présenta une chorégraphie sur Arksalalartôq de Micheline Coulombe Saint-Marcoux. 
36. Publié par la suite: "Le rôle du compositeur", Cahiers canadiens de musique, 11/12, 1975/1976.

37. Gervais, 1973 , p. 29-30 engagement dans le milieu en participant au comité d'organisation de la Semaine de musiques nouvelles présentée du 12 au 19 mai 1975.

Elle prend la parole, entre autres lors d'une table-ronde sur le thème des femmes et la création artistique, en compagnie de Marcelle Ferron, Jeanne Renaud et Yvette Brind'Amour, le 13 février 1975 à l'UQAM ; elle fait connâ̂tre la jeune création québécoise au Camp musical Orford en août $1975^{36}$ et au Festival de Guelph en mai 1976; elle présente une entrevue devant un public non initié à l'Institut Thomas More le 8 décembre 1977. En janvier 1979, elle est invitée au colloque consacré à la musique canadienne au Curso Latino Americano de musica contemporanea (Brésil); en mars 1981, devant le jeune public du Cégep de Saint-Laurent, elle précise sa position sur la place des compositrices dans la société; elle défend le rôle du compositeur dans une rencontre organisée par le ministère des Affaires culturelles en mai 1982. Elle raconte son propre parcours à Marcel Bélanger au cours d'une série radiophonique consacrée au travail de la création en 1982, ainsi qu’à Lyse Richer en 1984, entrevue qui sera gravée sur le coffret qui lui est consacré dans la série Anthologie de la musique canadienne produite par Radio Canada International.

Elle publie aussi quelques articles. L'entrevue qu'elle offre à Raymond Gervais en $1973^{37}$ mérite d'être soulignée car c'est le seul moment où elle met sur table les problèmes auxquels elle est confrontée depuis son retour d'Europe. Ainsi, rappelle-t-elle que le Conseil des Arts du Canada est demeuré sourd à ses nombreuses démarches pour financer un billet d'avion alors qu'elle tentait d'inviter le compositeur français Luc Ferrari au «Carrefour électroacoustique». De même, caressait-elle le projet de créer un événement autour de l'œuvre de François Bayle (monter Jeita ou le murmure des eaux en plein air dans une carrière dans l'est de Montréal) mais on l'adressera à la SMCQ, responsable, aux yeux des subventionnaires, de l'organisation de ce type de manifestation. Or, à l'époque, cette société musicale était encore assez traditionnelle dans les formules de concert, et ce projet fut refusé. Elle tente, sans succès, de convaincre Radio-Canada de diffuser des bandes qu'elle a rapportées de Paris, puis, revirement de situation, après le succès de l'événement "Carrefour électroacoustique », la société d'état en produit un disque, mais sans aucune référence à l'événement qui lui avait donné naissance. Elle soumet aux Jeunesses musicales du Canada le projet d'une tournée d'un programme contemporain conjointement avec la pianiste Christina Petrovska, mais la réponse fut si tardive que le projet dut être annulé. Elle profite enfin de cette entrevue pour témoigner sur la manière avec laquelle le chef d'orchestre Franz-Paul Decker a traité son œuvre pour 
orchestre, Hétéromorphie, présentée en 1970, refusant d'installer les instrumentistes dans différents endroits de la salle, comme l'exigeait la partition, pour les regrouper sur scène afin de lui faciliter la direction. L'effet fut désastreux. «Ce dernier fit tout pour saboter mon œuvre. Il la dirigeait n'importe comment. Après le concert, il m'a tourné le dos et s'est effacé en coulisse $3^{3}$.» D'autres textes traitent de sa démarche esthétique et proposent des réflexions sur l'approche multidisciplinaire de la création ${ }^{39}$, sur le parallélisme entre musique instrumentale et électroacoustique ${ }^{40}$ et sur le théâtre musical ${ }^{4}$.

Elle s'engage également dans l'administration. Membre du conseil du Centre de musique canadienne peu de temps après sa fondation à Montréal en 1973, elle ajoute à ses tâches une présence assidue au conseil de la SMCQ à partir de 1979 .

Ses premiers contacts avec la Société avaient été difficiles car non seulement devait-elle traverser le mur des préjugés et des non-dits des membres de la direction à l'égard d'une compositrice de «musique sérieuse », mais elle devait également tenter de modifier l'étiquette qu'on lui avait accolée depuis son retour de Paris: celle d'une compositrice identifiée à la musique électroacoustique, étiquette qui fournissait parfois l'occasion aux organismes subventionnaires de douter de la musique instrumentale ou mixte qu'elle soumettait. Rappelons aussi qu'à cette époque, les nouveaux compositeurs étaient parfois intimidés par une SMCQ fortement identifiée à l'Université de Montréal. Trois de ses professeurs siégeaient au conseil d'administration ${ }^{42}$ et plusieurs étudiants de cette institution collaboraient à l'organisation des concerts qui avaient lieu à la salle Claude-Champagne. Ce lien institutionnel disparaîtra lorsque la Société choisira en 1975 de se loger à la salle Pollack qu'elle quittera en 1992 pour trouver son installation définitive à la salle Pierre-Mercure.

Cela étant dit, Micheline a participé à l'aventure de la SMCQ et plusieurs de ses œuvres y furent jouées. De 1966 à 1986, année du concert hommage qui a suivi son décès, la SMCQ a présenté 24 œuvres de quatre compositrices (sur 641 œuvres): Barbara Pentland (1), Marcelle Deschênes (4) ${ }^{43}$, Norma Beecroft (5), Micheline Saint-Marcoux (14 dont 7 lors du concert hommage le 13 février 198644 ). Et ces choix n'ont rien à voir avec sa présence au conseil d'administration (elle en sera la vice-présidente durant trois ans), car durant ce mandat de 1979 à 1984, une seule œuvre y a été présentée. C'est donc dire que Micheline Coulombe Saint-Marcoux a ouvert le sentier pour les futures compositrices.

C'est à l'Atelier de recherche théâtrale de l'Eskabel, dirigé par Jacques Crète, qu'est créée sa dernière œuvre, Transit, le 3 octobre $1984^{45}$ dans une
38. Idem, p. 30.

39. Coulombe Saint-Marcoux, 1979, p. 4-6.

40. Coulombe Saint-Marcoux, 1984. 41. Jonassaint, 1984.

42. Jean Papineau-Couture, Serge Garant et Maryvonne Kendergi.

43. Talilalilalilalarequiem, 21 mars et 23 août 1974; MOLL, opéra lilliput pour six roches molles, 18 mars 1976 ; OPERAaaaAH, 10-11-12 mars 1983.

44. Ajoutons deux autres œuvres qui ont été jouées après son décès les 25 septembre 1986 et 19 janvier 1995 et nous avons le compte final : 16 œuvres de Micheline Coulombe SaintMarcoux ont été présentées par la SMCQ, parmi lesquelles Assemblages, 9 novembre 1971; Ishuma, 21 mars 1974, 17 et 19 novembre 1977; Genesis, 21 janvier 1977; Moments, $1^{\text {er }}$ octobre 1978; Mandala I, 11 novembre 1982 ; concert hommage, 13 février 1986 ; Miroirs, 25 septembre 1986; Horizons II, 19 janvier 1995. Nous remercions chaleureusement Réjean Beaucage de nous avoir permis de consulter sa base de données consacrée à la SMCQ.

45. L'œuvre sera ensuite diffusée à Radio-Québec le 22 janvier 1985 dans une réalisation modifiée de Guy Leduc. 
46. En mai 1978, la compositrice attend à l'aéroport d'Helsinki (Finlande) sa petite fille de 5 ans, Delphine, qui doit la rejoindre à 11 heures, mais celle-ci a manqué la correspondance à Copenhague et n'arrivera qu'en fin de journée. Toutefois, ce n'est que vers 15 heures que la mère apprend la raison du retard. C'est durant ces longues heures d'attente que, refaisant mentalement le parcours de sa vie, naît le premier scénario. Pour une analyse de cette œuvre, voir : Lévesque, 1991.

47. Table-ronde animée par Francine Larivée sur "La place des femmes dans la création artistique au Québec", UQAM, 13 février 1975. Notes manuscrites de Micheline Coulombe Saint-Marcoux. Archives de l'auteure. 48. Conférence sur "Les femmes créatrices", présentée le 3 mars 1981 au cégep de Saint-Laurent. Notes manuscrites de la compositrice. Archives de l'auteure.

49. De Chevigny, 1985 production de Pierre Barrat, directeur de l'Atelier lyrique du Rhin. Le sujet de la pièce part d'une expérience vécue ${ }^{46}$ qui aurait pu donner lieu à une réflexion sur la relation mère-enfant, mais la compositrice a choisi de donner une tout autre direction au projet. L'attente de sa petite fille dans un aéroport devient plutôt un prétexte pour réfléchir à sa propre vie de créatrice et aux choix qu'elle s'est imposés pour devenir une professionnelle dans le milieu artistique. Sur scène, deux personnages s'affrontent: «Elle», la compositrice, celle qui a fait le choix pleinement assumé d'une vie solitaire dans l'écriture, «l'Autre», une chanteuse rock, celle qu'elle aurait pu devenir au hasard de la vie. Écrit en étroite collaboration avec France Théorêt, ce théâtre musical peut être considéré comme l'aboutissement des recherches de Micheline Coulombe Saint-Marcoux sur la synthèse entre la voix, les instrumentistes, la bande magnétique, l'espace sonore et le jeu scénique. Mais il est surtout, selon moi, une réflexion sur la volonté inébranlable qu'a exigée l'élaboration de sa carrière professionnelle dans une époque parsemée d'embûches pour une femme, des difficultés qu'elle a souvent évoquées avant de les porter à la scène, comme en témoignent ces quelques commentaires:

Le milieu musical est un milieu extrêmement difficile, c'est un milieu de chapelles, de guerres, de luttes. Il s'agit d'être tenace et de foncer. Si la porte ne veut pas s'ouvrir, on [escalade] le mur. Ne pas renoncer à ses projets, mais aussi refuser de se laisser embrigader dans un système périmé où la femme a la responsabilité des enfants et où la femme se sent coupable de déléguer ses pouvoirs. Moins d'enfants parce que la famille telle qu'elle existe (élément essentiel de notre société) ne favorise pas la femme. La femme doit faire un choix. Si elle refuse de participer à perpétuer le système, elle possède l'arme $n^{\circ} 1$ de révolution. Si l'on veut qu'elle continue de procréer, que l'on trouve des formules de rechange qui l'aideront à élever ses enfants ${ }^{47}$.

«Même s'il y a une certaine forme de discrimination, je préfère poursuivre mon chemin, ne pas me laisser décourager par cet état de fait et orienter mon énergie, à concentrer mon action sur le vrai problème: celui de la création $4^{8}$.»

«La société n'est pas prête à accueillir les femmes qui font une carrière et qui sont en même temps mère et épouse. La maternité devient une expérience fantastique une fois que l'on passe par-dessus la culpabilité que les autres essaient de nous imposer ${ }^{49}$.»

Micheline nous a quittés le 2 février 1985 à l'âge de 46 ans après une longue lutte contre le cancer.

Le parcours de Marcelle Deschênes est tout à fait différent. En marge du milieu officiel depuis son retour d'Europe, elle s'intéresse avant tout à l'art de 
la performance et des happenings, courants artistiques américains créés en réaction à l'hyper-intellectualisme de la musique européenne, ainsi qu'à la nouvelle poésie orale qu'elle découvre dans les spectacles de Raoul Duguay. C'est dans cet esprit qu'elle produit en mars 1972 Si... longtemps, un événement multimédia de 15 heures sur la note si offert aux visiteurs des Portes ouvertes de la Faculté de musique de l'Université de Montréal $5^{\circ}$.

Tout en gagnant sa vie comme accompagnatrice de quelques chansonniers, elle se joint au GIMEL et obtient une charge de cours à l'Université Laval. Elle y enseignera de 1972 à $1978^{51}$. Durant cette période, elle s'investit dans la recherche en animation multidisciplinaire et organise, en collaboration avec le compositeur Richard Martin et le mime Robert Séguin, des sessions de création collective pour des groupes de non-musiciens. Ces expériences la conduisent à une longue réflexion sur les mécanismes de la perception auditive. C'est durant ce séjour à Québec qu'elle compose MOLL, opéra lilliput pour six roches molles, pour trombone, clarinette, percussions et jouets d'enfant, une commande de la SMCQ qui sera créé le 18 mars $197^{5^{2}}$. Il faut contempler la partition pour saisir toute la dimension multidisciplinaire de cette pièce. Marcelle Deschênes trouve ici son langage personnel. «C'est une coupure radicale avec l'écriture sérielle devenue stérile pour moi », note la compositrice dans son journal. «Je dois tout faire pour m'en échapper! et tenter d'expérimenter d'autres modes d'organisation des sons plus inspirants que des séries de chiffres, ouvrant davantage sur une pluralité de niveaux en connexion avec la perception, le référentiel, les archétypes, les modèles, etc. ${ }^{33}$ » Ce référentiel, elle le trouve dans l'univers de l'enfance. «Dans cette suite de 17 miniatures, écrit-elle, j’ai voulu y exprimer la révélation d'un itinéraire à travers la lente usure du temps. Explorer l'envers des choses menues, faire une introspection du monde de l'enfance, le petit immensément grand, la dureté se transmuant en mou, en tendre, en flexible, l'inerte en vivant, le drame en merveille, la tristesse en folie douce ${ }^{54}$. » Une vraie révélation au public de la SMCQ. Cette œuvre lui vaudra le premier prix de musique mixte au $6^{\mathrm{e}}$ Concours international de musique électroacoustique de Bourges en mai 1978 et un Euphonie d'Or au $20^{\mathrm{e}}$ concours de Bourges en 1992.

L'engagement de Marcelle Deschênes dans le milieu prend une nouvelle voie lorsqu'elle quitte Québec pour s'installer à Montréal et fonder son propre studio, Bruit Blanc. Elle devient membre d'un groupe de jeunes compositeurs (Yves Daoust, Michel Longtin, Philippe Ménard, Jean Sauvageau et Pierre Trochu) et participe avec eux à la fondation de l'Association pour la création et la recherche électroacoustique du Québec (ACREQ)55,
50. Ce qui n'est pas sans rappeler les Dream Houses de La Monte Young créées en 1963.

51. C'est au cours des concerts du GIMEL que le compositeur Robert Normandeau découvrira Marcelle Deschênes avant qu'elle ne devienne sa professeure à l'Université de Montréal en 1984.

52. Elle en fera une version électroacoustique l'année suivante. On peut entendre cette œuvre ainsi que Lux, Indigo, Le bruit des ailes et Big Bang I/ et I/I sur un enregistrement récent produit par Empreintes DIGITALes et diffusé par IMED 0681, qui a valu à la compositrice et à son diffuseur le prix Opus 2006-2007.

53. Archives personnelles de Marcelle Deschênes.

54. Site Marcelle Deschênes sur <http :// www.electrocd.com>

55. On ne dira jamais assez combien cette association a contribué à l'essor de ce nouveau langage musical, grâce, entre autres, et dès 1978, à de nombreuses activités de diffusion de concerts acousmatiques dans des lieux très diversifiés et à son réseau international, particulièrement son association au Festival de Bourges. II n'y manquait qu'un organisme pour assurer la pérennité de ces œuvres: une compagnie de disques. Ce sera le travail du compositeur Jean-François Denis qui, après avoir créé la magnétothèque à l'Université Concordia, publie un premier catalogue, et fonde avec Claude Schryer en 1989 la compagnie de disques Empreintes DIGITALes. 
56. Cette œuvre valut à son auteure et à Jacques Collin, concepteur des images, le Silver Award par le AMI International Multi-Image Competition of California en 1986. association qui sera étroitement liée à la création d'un nouveau projet multidisciplinaire: un opéra.

La présentation durant trois jours, à guichets fermés, les 10, 11 et 12 mars 1983, à la salle Marie Gérin-Lajoie de l'UQAM du spectacle multimédia OPERAaaaAH a eu l'effet d'une bombe dans le milieu musical. Produit par l'ACREQ et la SMCQ, avec la collaboration de Raoul Duguay, des Mimes électriques, du groupe Performance Multi-Média, Jacqueline Lemieux, Denis Latendresse, Jean Corriveau et Alain Thibault, jamais un tel événement, d'une ampleur «wagnérienne ", n'a suscité autant de discussions à l'époque. Et pour cause. L'auteure nous livrait sa vision du monde. Le public assistait à une genèse imaginaire de l'univers dans laquelle l'auteure avait inscrit son univers psychique et ses préoccupations sociales: une synthèse artistique où se côtoyaient simultanément la peinture lumineuse tridimensionnelle sur corps humain (un écran humain, conçu par l'artiste Paul Saint-Jean), la projection de diapositives synchronisée avec la bande magnétique, la pantomime, une performance de Monty Cantsin qui procédait en direct à une prise de sang sur son propre corps, des odeurs de fraises et de foin mouillé, le tout accompagné d'une diffusion musicale que traversait, telle une lame acérée, un fragment du Requiem de Mozart et d'autres citations. L'œuvre comprenait cinq tableaux dont certains ont été par la suite diffusés de manière autonome. Les deux plus connus demeurent aujourd'hui L'Écran humain et Jour 'J' où l'auteure aborde les préoccupations écologiques et la destruction de la nature. Mais jusqu'où ira la colère de Dieu?

Dans deUS irae, composé en 1985, Marcelle Deschênes s'inspire de deux romans de science-fiction, Le carnaval de fer de Serge Brussolo et de Deus Irae de Philip K. Dick et Roger Zelazny. Le premier relate l'histoire d'un homme qui tente de retracer l'itinéraire d'un pèlerinage interdit par la loi et qui prend l'allure d'un dangereux jeu de piste, alors que le second raconte une vision d'un voyage halluciné sur une terre dévastée, après l'holocauste où de rares survivants vénèrent le dieu de la colère, celui qui a semé l'horreur atomique. L'auteure met en scène un couple en fuite et un enfant impuissant devant ce même dieu de la colère, ce père vengeur, maître de la parole (représenté par l'aigle américain) qui étend les pluies acides à la terre entière. Ce fut un spectacle bouleversant ${ }^{6}$, un vibrant témoignage sur notre époque, et je me suis longtemps demandé comment un tel cumul de colère et de révolte pouvait exister dans la tête d'une femme par ailleurs si timide et si réservée. Marcelle Deschênes en donne la réponse: 
Comme dans la musique sorcière sans doute impure (!), je veux d'abord promouvoir les valeurs non officielles que les détours culturels et politiques nous ont contraints à abandonner et ainsi revenir à l'écoute de nos gestes, nos regards, nos mains, nos ventres, nos lèvres, nos odeurs, nos passions, nos sons, nos paroles, nos rires, nos cris, notre imagination pour retrouver la dimension perdue. Et tant mieux si cette grande part d'illogisme nous conduit à une musique qui n'est dans rien mais se sert de tous les langages dans la plus grande liberté 57.

Pour celle qui n'a presque pas offert d'entrevues ou publié quelque article sur son cheminement artistique, jamais credo ne fut exprimé avec autant de force et de revendication.

\section{Un programme... enfin}

Tout comme Micheline Coulombe Saint-Marcoux dans Transit, le parcours de Marcelle Deschênes, dans cette œuvre et celles qui ont suivi $5^{8}$, marque un retour à la musique «à programme », hautement référentielle et subjective, contribuant ainsi à l'émergence d'une esthétique postmoderne déjà inscrite dans les dernières créations musicales de Claude Vivier. Elle a également ouvert la voie au début des années 1980 à la mise sur pied d'un nouveau programme en composition électroacoustique à l'Université de Montréal (aux trois cycles) où l'orientation multimédia est devenue, depuis l'engagement en 1988 du compositeur Jean Piché, une voie privilégiée. Mais, à ses débuts, et jusqu'à la mise en marché des logiciels informatiques, la formation proposée par Marcelle Deschênes visait essentiellement la musique sur bande magnétique, une orientation «acousmatique» dont le compositeur français Francis Dhomont59 se fera plus tard l'ardent défenseur en établissant les bases de son discours esthétique. Elle intégra à ce programme un cours qui avait été créé quelques années auparavant par deux compositrices, Ginette Bellavance et Hélène Prévost: «perception auditive », un apprentissage alternatif au traditionnel cours de solfège, inspiré du Traité des objets sonores de Pierre Schaeffer. Nommé chargé de cours en 1984, Dhomont prendra éventuellement la relève de ce cours ${ }^{6 \circ}$ et enseignera la composition complémentaire et les techniques d'écriture électroacoustique, autre cours créé par Marcelle Deschênes.

Selon les souvenirs de Robert Normandeau ${ }^{61}$, Marcelle a toujours défendu une pédagogie entièrement centrée sur le développement de la personnalité artistique des candidats plutôt que sur la transmission d'une pensée esthétique à sens unique, une attitude qui provenait certainement de son souvenir « du temps des corsets ». Pour Francis Dhomont par contre, il s'agissait davantage d'imposer dans le milieu une nouvelle manière de penser la création
57. Programme de la SMCQ, $143^{\mathrm{e}}$ concert, $17^{\mathrm{e}}$ saison, 1982-1983. 58. Parmi lesquelles: Lux (1985), une musique qui accompagne un vidéoclip tridimensionnel géant conçu par Renée Bourassa; noUS, D-503 NOUs, création radiophonique à partir du roman WE d'Eugène Zamiatine (1920) dont s'est inspiré George Orwell pour son roman 1984; Big Bang // (1987), installation multimédia en collaboration avec Georges Dyens; Ludi (1990), opéra médiatique écrit en collaboration avec Renée Bourassa; Big Bang III (1993), installation multimédia; Le bruit des ailes (2002), musique acousmatique.

59. Francis Dhomont (Paris, 1926) s'est installé à Montréal en 1978 après avoir épousé Marthe Forget, professeure de chant à la Faculté de musique de I'Université de Montréal et fondatrice de l'Atelier de jeu scénique. Elle a prêté sa voix à la première œuvre de Dhomont, Sous le regard d'un soleil noir, composée entre 1979 et 1981.

6o. Francis Dhomont note à propos de ce cours: "Initiated by M. Deschênes, continued by F. Guérin, systematized at first by F. Dhomont and now by R. Normandeau and S. Roy", dans Dhomont, 1996 (p. 26, note 9). Qu'est-ce qui se cache derrière cette expression "systématisé en premier"? Qu'il a érigé en système ce qui lui semblait auparavant non organisé? Ou, ce qui serait plus juste, qu'il a adapté à son mode de pensée un contenu de cours qui lui a été confié?

61. "Marcelle Deschênes avait une oreille formidable, une manière de permettre aux étudiants de découvrir leur propre langage, de progresser à leur manière dans le monde de l'inouï" (témoignage de Robert Normandeau [Sforzando, revue de l'Association des diplômés de la Faculté de musique de I'Université de Montréal, 8/1, printemps 2001, p. 6]. 
62. Idem, p. 27. En 2004, un certain animateur radiophonique français, Bernard Girard, proposa une série d'émissions où, sous le titre "Francis Dhomont et l'école de Montréal ", il magnifia le rôle de Dhomont jusqu'à devenir "le père de l'école de Montréal " qui, après avoir obtenu "une chaire en composition " à l'Université de Montréal, aura formé plus de 200 musiciens. Ce sont les distorsions de I'Histoire qui se répètent. Dommage... Les textes de ces émissions sont disponibles sur internet.

63. Bien que transmise par "chuchotement", la réaction de certains compositeurs à cette "école" n'est pas sans rappeler la querelle littéraire de 1947 où Robert Charbonneau, dans La France et nous, réagissait à la dépendance intellectuelle des Canadiens français à l'égard de la France exprimée par l'écrivain Georges Duhamel qui considérait la littérature québécoise comme "une branche de l'arbre français". Voir Nardout-Lafarge, 1993.

64. Une des solutions à ces problèmes est venue des femmes elles-mêmes, d'une nouvelle génération, dans l'initiative que certaines ont prise, en 1996, de fonder, à l'extérieur des institutions d'enseignement, leur propre centre d'apprentissage, "un studio à soi ". Le Studio XX est en effet devenu un lieu qui leur permet de réfléchir à la situation des femmes dans ce milieu, de s'initier aux nouvelles technologies à leur rythme et de façonner le cyberespace à leur manière afin de créer et de diffuser un univers multimedia qui parle d'elles. Entièrement autogéré, le Studio XX présente annuellement le festival HTMlles, diffuse une émission radiophonique hebdomadaire et organise des cours d'apprentissage de logiciels spécialisés. Depuis huit ans, plus de 300 femmes ont participé à ces séances de formation en création multimédia. Hélène Prévost, réalisatrice exceptionnelle de Radio-Canada électroacoustique à laquelle il donnera éventuellement le nom d'«école de

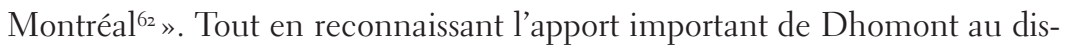
cours sur la musique électroacoustique au Québec, on doit souligner ici que cette appellation a créé un malaise. Certains compositeurs, inclus dans cette "école», ont été réfractaires à cette étiquette qui leur a été accolée, alors que d'autres, exclus ou ignorés, réclamaient que l'on reconnaisse le rôle qu'ils avaient joué dans le développement de la musique acousmatique. Si l'expression est courante en France où le sens de la hiérarchie est beaucoup plus ancré dans la culture, elle irrite certains au Québec où l'esprit individualiste et indépendant a historiquement imprégné fortement les comportements et les attitudes ${ }^{63}$.

Cependant, malgré l'importance qu'ont eue Micheline Coulombe SaintMarcoux et Marcelle Deschênes dans l'élaboration des structures qui ont conduit à la reconnaissance de ce nouveau médium d'expression musicale, très peu de femmes ont, depuis, choisi cette voie institutionnelle. Car, contrairement aux programmes d'enseignement de la composition instrumentale où les étudiantes ont pu trouver chez certains compositeurs un terrain propice à la création (tel qu'il a été expliqué précédemment), le milieu électroacoustique (art acousmatique ou art médiatique) a été et demeure encore aujourd'hui un lieu presque essentiellement masculin. Est-ce à cause d'un blocage psychologique devant l'apprentissage des technologies? Ou encore, à cause de l'absence d'une réflexion pédagogique sur la manière de transmettre ces compétences aux femmes? Ou d'un environnement masculin résistant à la présence des femmes ${ }^{64}$ ?

Pour les femmes de ma génération, l'arrivée de l'ordinateur personnel au début des années 1980 a eu lieu au moment où nous atteignions la jeune quarantaine et, pour plusieurs, vivions une situation monoparentale. Le temps requis pour apprivoiser ce nouvel environnement technologique qui se modifiait sans cesse nous faisait défaut. Interpellées par les tâches professionnelles et familiales, on ne pouvait passer de six à huit heures consécutives (comme on le voit couramment aujourd'hui) à se familiariser avec le «maléfique engin» et l'impatience que manifestait l'entourage masculin devant nos difficultés à saisir rapidement le fonctionnement de cette «machine » explique la rareté des compositrices en ce domaine à la fin du $\mathrm{xx}^{\mathrm{e}}$ siècle ${ }^{65}$. Ce sont les difficultés auxquelles ont été confrontées Micheline Coulombe Saint-Marcoux et Marcelle Deschênes qui, malgré ces handicaps, et parce qu'elles voulaient sortir des sentiers battus, ont compris que cet outil pourrait servir à exprimer musicalement une nouvelle sensibilité artistique. 


\section{B I B LIOGRAPHIE}

BeAucage, Réjean (2008), «Schaeffer au Québec en quelques allers-retours », dans: Gayou, Évelyne (éd.) Pierre Schaeffer: portraits polychromes, Paris, INA, p. 19-23.

Borvin, Jean (en collaboration avec Patrick Hébert), (2001) «Trois œuvres de musique québécoise marquantes diffusées dans les pavillons lors de l'Exposition universelle de Montréal en 1967", Les Cahiers de la Société québécoise de recherche en musique, 5/1, p. 75-90.

ChÉnARd, Ginette (2008), «Le Québec à l'ère de la mondialisation: tradition et modernité, un regard croisé harmonieux», dans Weidmann-Koop, Marie-Christine (éd.), Le Québec à l'aube du nouveau millénaire: entre tradition et modernité, Québec, Presses de l'Université du Québec, p. 48-56.

Coulombe Saint-Marcoux, Micheline (1968), «Réflexions d'une jeune compositrice», Vie musicale, mai, p. 13-16

Coulombe Saint-Marcoux, Micheline (1979) «Musique et arts visuels: l'œil écoute», Cahiers [Cahiers des arts visuels au Québec, Sherbrooke], nº 4, p. 4-6.

Coulombe Saint-Marcoux, Micheline (1984), « Au carrefour de deux esthétiques », Spirale, $\mathrm{n}^{\circ} 40$, février, p. 10.

De Chevigny, Marie-Claude (1985), «Le son dans les veines », Châtelaine, janvier, p. 58.

Deschênes, Aldégonde, (1993) Quatre saisons dans la classe d'Aldégonde, Salon de littérature de Mont-Joli.

Dhomont, Francis (1996), «Is there a Québec Sound?», Organized Sound, vol. 1, n 1, p. $23-28$.

GanN, Kyle (2008), "Que signifie et ne signifie pas la musique des femmes? », article dans ce numéro.

Gervais, Raymond (1973), TILT [Département d'Histoire de l'art de l’UQAM], décembre, p. 29-30.

Green, Anne-Marie et Ravet, Hyacinthe (éd.) (2005), L'accès des femmes à l'expression musicale, Paris, L'Harmattan.

JonASSAINT, Jean, (1984), «Écrire un théâtre musical. Transit. Entretien avec Micheline Coulombe Saint-Marcoux et France Théoret», Dérives, n ${ }^{\circ s} 44-45$, p. 74-84.

Lefebvre, Marie-Thérèse (2004), Rodolphe Mathieu (189o-1962): l'émergence du statut professionnel de compositeur au Québec, Québec, Éditions du Septentrion.

LÉvesque, Carole (1991), «Transit de Micheline Coulombe Saint-Marcoux: l'aboutissement du cheminement du créateur », Les Cahiers de l'ARMuQ, nº 13, p. 78-89.

Nardout-Lafarge, Élisabeth (1993), « Histoire d'une querelle», dans: Charbonneau Robert (éd.), La France et nous, Montréal, Bibliothèque québécoise, p. 7-26.

NAtтiEz, Jean-Jacques (1987), «Y a-t-il une musique québécoise? », InHarmoniques, nº 2, p. 152160 .

qui s'est vouée durant vingt ans à la diffusion radiophonique des musiques nouvelles, en est maintenant la viceprésidente.

65. Ce qui explique aussi la raison pour laquelle aucune compositrice québécoise n'a participé à la rédaction des trois numéros de la revue Circuit consacrés à l'électroacoustique (vol. 4, $n^{\circ} 1 / 2,1993$; vol. 13, $n^{\circ} 1,2002$; vol. 13, $n^{0} 3$, 2003). Seule Françoise Barrière, compositrice française, y a publié un article. 